\title{
Variables que afectan la satisfacción con la alimentación según nivel socioeconómico: un estudio exploratorio en el sur de Chile
}

\author{
Variables affecting food satisfaction \\ according to socioeconomic status: \\ an exploratory study in southern Chile
}

\begin{abstract}
In order to identify variables that influence in food satisfaction for people belonging to different socioeconomic status (SES) in southern Chile, a survey was administered to a sample of 400 people living in the major cities of this region. The questionnaire included the SWFL (Satisfaction with Food-related Life) scale and the IQLRH (Index of Quality of Life Related to Health). Demographic characteristics and dietary habits were also consulted. Four ordinal logit models were proposed, where the dependent variable was satisfaction with food-related life, considering the total sample and three subsamples: medium and medium-high strata (ABC1), medium-medium (C2) and medium-low (C3), low (D) and very low (E) as a whole. The four models were significant $(p<0.001)$, but differed in the explanatory variables that were significant. Regardless of SES, satisfaction with food-related life decreases if the person does not have a good perception of their own health. Other variables that influence satisfaction with food-related life, which differed according to the SES, are related to the gender, the family composition, the time available for family dining, frequency of consumption of meat and use of fast food outlets and the restriction of alcohol consumption.
\end{abstract}

Key words: Satisfaction with food-related life, socioeconomic status, health.
Berta Schnettler M. (1)

Horacio Miranda V. (1)

Ligia Orellana C. (2)

José Sepúlveda M. (2)

Marcos Mora G. (3)

Germán Lobos A. (4)

(1) Departamento de Producción Agropecuaria, Facultad de Ciencias Agropecuarias y Forestales, Universidad de La Frontera, Temuco, Chile. (2) Centro de Psicología Económica y del Consumo. Universidad de La Frontera, Temuco, Chile. (3) Departamento de Economía Agraria, Facultad de Ciencias Agronómicas, Universidad de Chile, Santiago, Chile. (4) Escuela de Ingeniería Comercial, Facultad de Ciencias Empresariales, Universidad de Talca, Talca, Chile.

Dirigir la correspondencia a: Profesora

Berta Schnettler Morales Avenida Francisco Salazar 01145 Casilla 54-D Temuco. Chile. Teléfono: 56-45-2325655

E-mail: berta.schnettler@ufrontera.cl

Este trabajo fue recibido el 25 de Enero de 2014 y aceptado para ser publicado el 5 de Mayo de 2014.

\section{INTRODUCCIÓN}

La satisfacción con la alimentación se define como la evaluación general que hace la persona respecto a su alimentación (1). Estudios recientes reportan una relación positiva entre la satisfacción vital (componente cognitivo del bienestar subjetivo) y la alimentación como dominio de la vida, de forma que aquellas personas que están satisfechas con su alimentación se encuentran satisfechas con su vida (1-5). Aunque aún existen pocas investigaciones enfocadas en estudiar las variables que se relacionan con la satisfacción con la alimentación, se ha determinado que se asocia con la preferencia hacia ciertos alimentos $(6,7)$, con comportamientos alimentarios saludables (6-9), con la interacción familiar y social en torno a la alimentación $(3,8-10)$, con la capacidad de disfrutar los alimentos y el significado hedónico que estos tienen (8-10) y, con una mejor salud física (10) y mental (3). También se ha detectado relación entre la satisfacción con la alimentación y algunas características demográficas, como la edad $(6,7,9)$, la composición del grupo familiar y $(6-8)$, el estado civil $(7,8)$ y el género (8). El nivel socioeconómico (NSE) de la persona también se relaciona con la satisfacción con la alimentación (9). Al respecto, la satisfacción con la alimentación se asocia con la disponibilidad de recursos económicos para gastar en alimentación $(7,8)$, lo que además repercute positivamente en un mayor nivel de satisfacción con la vida de las personas (2).

Es indiscutible que la alimentación es un requisito para que las personas estén conformes con sus vidas. La falta de alimentos genera descontento (1). Estudios llevados a cabo con muestras de personas de bajos ingresos en Etiopía y Perú, indican que la disponibilidad de suficientes alimentos constituye una de las condiciones fundamentales para tener una buena vida $(11,12)$. Las personas de bajos ingresos poseen una nutrición inadecuada que incrementa la probabilidad de enfermedades (13). Numerosos estudios indican que la posibilidad 
de gastar una mayor cantidad de dinero en alimentos para el hogar, se asocia con la posibilidad de acceder a alimentos de mayor calidad y más saludables (14-17). Al respecto, cuando las personas tienen acceso a una suficiente oferta alimentos, es esperable que estos no sean tan relevantes para las personas respecto de aquellos que viven en condiciones de restricción. Sin embargo, las consideraciones y actividades diarias en torno a la obtención, preparación y consumo de alimentos constituyen una importante preocupación para las personas. Por tanto, a pesar de que exista suficiente disponibilidad de alimentos, la alimentación igualmente ocupa una parte importante de la vida de las personas, en términos de tiempo y recursos (1), aspectos de salud asociados a la alimentación (3, 6-10), interacción familiar y social $(3,8-10)$ y de placer (8-10). El objetivo de la presente investigación fue identificar variables que influyan en la satisfacción con la alimentación en personas pertenecientes a distintos NSE en las principales ciudades del sur de Chile, a través de la aplicación de un modelo logit ordinal.

\section{SUJETOS Y MÉTODOS Sujetos}

En esta investigación de tipo exploratoria, se aplicó un cuestionario estructurado en forma personal a una muestra de 400 personas de las principales ciudades de las Regiones del Biobío, La Araucanía y Los Lagos en el sur de Chile. El tamaño muestra se obtuvo mediante la fórmula de muestreo aleatorio simple para poblaciones no finitas $(\mathrm{N}>100.000)$, considerando $95 \%$ de confianza y $5 \%$ de error de estimación con p y q de 0,5 (18). Las encuestas fueron realizadas en las ciudades con más de 100.000 habitantes de estas regiones, las cuales fueron distribuidas en forma estratificada con afijación proporcional en Chillán (60 encuestas), Concepción (189 encuestas), Temuco (91 encuestas) y en Puerto Montt (60 encuestas). La encuesta fue aplicada al azar por un encuestador previamente entrenado (psicólogo) en centros comerciales con supermercado y patio de comida, ubicados en distintas zonas socioeconómicas de las ciudades en estudio, entre julio y agosto de 2012. Previo a esto se realizó la validación del cuestionario mediante un pretest con el 5\% de la muestra. La realización del estudio fue aprobada por el Comité de Bioética de la Universidad de La Frontera. Los participantes firmaron consentimientos informados previo a la aplicación del cuestionario.

\section{Instrumento}

El cuestionario aplicado incluyó las siguientes escalas: Satisfaction with Food-related Life (SWFL), que fue propuesta y probada por Grunert et al. (1) en ocho países europeos, mostrando adecuados niveles de consistencia interna y la existencia de una sola dimensión que agrupa los cinco ítems de la escala: 1. Los alimentos y comidas son elementos muy positivos en mi vida. 2. Yo estoy muy complacido con mi alimentación. 3. Mi vida en relación a los alimentos y comidas se acerca al ideal. 4. Respecto a los alimentos, mi condición de vida es excelente. 5. Los alimentos y comidas me proporcionan gran satisfacción en mi vida diaria. El encuestado debió responder su grado de acuerdo con cada una de estas afirmaciones mediante una escala tipo Likert de 6 niveles (1: completamente en desacuerdo, 6: completamente de acuerdo). La SWFL ha mostrado adecuados niveles de consistencia interna ( $\alpha$ de Cronbach: 0,82-0,88) en estudios previos en Chile (5-8).

Índice de Calidad de Vida Relativo a la Salud (ICVRS): desarrollado por Hennessy et al. (19), consta de cuatro ítems que exploran la auto percepción de la salud, salud física reciente (enfermedades físicas y heridas), salud emocional reciente (estrés, depresión y problemas emocionales) y limitaciones recientes en la actividad (trabajo, estudios o recreación).

Se consultó la frecuencia de consumo de algunos de los alimentos adaptados de los grupos de gasto que utiliza el Instituto Nacional de Estadísticas (INE) en las Encuestas de Presupuestos Familiares (20): pan, carne (bovina, pollo, cerdo, entre otros), leche y huevos, frutas, verduras y vino. También se consultó si alguno de los siguientes productos se restringe en el hogar por motivos de salud o prevención: azúcar, sal, grasas y frituras y alcohol. Se preguntó la frecuencia de comidas en el hogar junto a la familia y el tiempo disponible para esto $y$, la frecuencia de comidas fuera del hogar en restaurantes y locales de comida rápida. Se incluyeron preguntas de clasificación de los encuestados: género, edad, estado civil, número de integrantes de la familia, zona de residencia, ocupación y estudios del jefe de hogar y, la tenencia de 10 bienes domésticos. Estas dos últimas variables permiten determinar el nivel socioeconómico (NSE) correspondiente a ABC1 (alto y medio alto), C2 (medio-medio), C3 (medio-bajo), D (bajo) y E (muy bajo) (21).

\section{Análisis estadístico}

El análisis de los resultados se realizó con el programa SPSS 16.0 en español para Windows. La extracción de factores de las escalas se realizó con análisis factorial de componentes principales considerando autovalores mayores que 1 (22). La consistencia interna de las escalas fue calculada usando el coeficiente $\alpha$ de Cronbach (22). Con el objeto de contrastar la influencia de distintas variables explicativas en la satisfacción con la alimentación en personas de distinto NSE en las principales ciudades de la zona centro sur de Chile, se plantearon cuatro modelos logit ordinales (23), en los que se introdujeron como variable dependiente el nivel de satisfacción con la alimentación, y como variables explicativas las respuestas obtenidas en el cuestionario aplicado. Las cuatro especificaciones del modelo logit ordinal correspondieron a la muestra total y por separado se realizaron las regresiones correspondientes a los NSE ABC1 (alto y medio-alto, $n=120$ ), C2 (medio-medio, $n=$ 160) y en conjunto a los grupos C3, D y E (medio-bajo, bajo y muy bajo, $n=120$ ). Estos tres últimos grupos se tomaron en conjunto debido al bajo número obtenido en cada categoría por separado. Los parámetros de los modelos logit fueron estimados por el método de máxima verosimilitud (23). La especificación de las variables dependiente y explicativas que resultaron significativas en los modelos generados se presentan en la tabla 1. Se utilizaron las siguientes medidas de la bondad de ajuste del modelo: $R^{2}$ adj de Nagelkerke (Pseudo- $R^{2}$ ) y -2 log de verosimilitud. Para medir la significancia estadística de las variables explicativas se utilizó el estadístico Wald.

\section{RESULTADOS}

La muestra total estuvo compuesta en mayor proporción por mujeres $(52,1 \%)$, con edades entre 31 y 45 años $(38,7 \%)$; solteros, viudos, divorciados o separados $(53,2 \%)$, residentes en una zona urbana $(90,8 \%)$, pertenecientes a grupos familiares con tres a cuatro integrantes $(55,7 \%)$, con estudios universitarios $(45,9 \%)$ y que se desempeñan como empleados $(66,6 \%)$.

La escala SWFL mostró un adecuado nivel de consistencia interna ( $\alpha$ de Cronbach $=0,993$ ) y la existencia de un único factor que agrupó los cinco ítems que componen la escala con el $76,6 \%$ de la varianza explicada. Las categorías "extremadamente insatisfecho" e "insatisfecho" fueron consideradas en conjunto, debido al bajo número de personas en 
cada una. De esta forma, en la muestra total el $28,8 \%$ estuvo extremadamente satisfecho; $43,0 \%$ satisfecho; $20,6 \%$ medianamente satisfecho y $10,1 \%$ insatisfecho con su alimentación. Mediante la prueba $\mathrm{Chi}^{2}$ se obtuvo superior proporción de personas extremadamente satisfechas con su alimentación en el NSE ABC1 (37,0\%) y mayor presencia de insatisfechos en la submuestra formada por los NSE C3, D y E $(22,7 \%)(P=0,004)$. EL ICVRS mostró un adecuado nivel de consistencia interna ( $\alpha$ de Cronbach $=0,755$ ) para tres de los cuatro ítems, los que se agruparon en único factor con el 61,3\% de la varianza explicada. En la primera pregunta del ICVRS, la mayor proporción de encuestados tuvo una auto percepción muy buena $(41,8 \%)$ y buena $(32,6 \%)$ de su salud. Mediante la prueba de Chi2 se observó mayor proporción de personas pertenecientes al NSE ABC1 $(53,4 \%)$ que tiene una auto percepción muy buena de su salud, mientras en la submuestra formada por los NSE C3, $D$ y $E$ fue mayor la proporción de personas que tuvo una auto percepción regular $(22,7 \%)$ de su salud $(P=0.000)$. El promedio de días totales con problemas de salud o limitaciones en las actividades habituales por este tipo de problemas en los últimos 30 días fue de 6,49 (DS=3,71) en la muestra total. El promedio de días correspondiente a la submuestra formada por los NSE C3, D and E fue estadísticamente superior al promedio del NSE ABC1 $(P=0,026)$.
Respecto a los hábitos de consumo de alimentos en el hogar en la muestra total, fue mayor el consumo diario de pan $(84,5 \%)$, dos a tres veces por semana de carne $(51,9 \%)$, y diario de leche y huevos $(58,5 \%)$, frutas $(51,6 \%)$ y verduras (56,3\%). La mayor proporción de participantes no consume vino $(43,0 \%)$ y si lo hace, corresponde a consumo ocasional (35,4\%). Utilizando la prueba de $\mathrm{Chi}^{2}$, se detectaron diferencias significativas sólo en el consumo de leche y huevos $(P=0,001)$, debido a que una mayor proporción de participantes pertenecientes al NSE ABC1 indicó consumir estos productos en forma diaria $(67,7 \%)$, mientras que en la submuestra formada por los NSE C3, D y E fue superior la presencia de personas que los consume una vez por semana $(10,7 \%)$ o sólo ocasionalmente (5,3\%). Alrededor del 37\% de los encuestados indicó restringir el consumo de azúcar y sal, mientras que en torno al $45 \%$ restringe el consumo de grasas y frituras y vino, sin diferencias significativas $(P>0,1)$ entre las submuestras. El $64,5 \%$ de la muestra total come en restaurantes ocasionalmente o casi nunca, el $69,3 \%$ respondió que nunca o sólo ocasionalmente come en locales de comida rápida. Fue significativamente superior $(P=0,009)$ la proporción de personas del NSE ABC1 que generalmente $(12,8 \%)$ y ocasionalmente $(40,6 \%)$ come en restaurantes, mientras que los participantes de la submuestra C3, D y E en mayor proporción nunca lo hace (37,3\%). La

\section{TABLA 1}

Definición de variables dependientes y variables explicativas para el modelo de regresión logit ordinal generado para medir la satisfacción con la alimentación en personas de la zona Centro Sur de Chile.

Variable Dependiente

Satis-Alim

Variables explicativas

Per-Salud

Frec-Com-Rap

Gén

Res-Alcohol

Tiempo-Cena-Sem

Miem-hogar

Frec-Carne

\section{Descripción}

Satisfacción con la alimentación (multinomial): $<<1>>$ implica que la persona declara estar insatisfecho con la alimentación, $<<2>>$ implica que se declara medianamente insatisfecho, $<<3>>$ satisfecho, $<<4>>$ extremadamente satisfecho.

\section{Descripción}

Percepción de Salud (multinomial): $\langle<1>>$ significa que la persona considera que su salud en general es muy mala y regular, $<<2>>$ buena, $<<3>>$ muy buena, $<<4>>$ excelente.

Frecuencia con que el encuestado come en locales de comida rápida (multinomial): $<<0>>$ implica que la persona nunca come en locales de comida rápida, $<<1>>$ significa que la persona come casi nunca (1 ó 2 días por año) en locales de comida rápida, $<<2>>$ cuando la persona lo hace ocasionalmente (1 ó 2 días por mes), <<3>> generalmente (1 ó 2 días por semana), <<4>> siempre (varios días por semana).

Género (binomial): $<<1>>$ significa que la persona es del género masculino, $<<2>>$ es del género femenino.

Restricción del alcohol en el hogar por motivos de salud o prevención de enfermedades (binomial): $<<0>>$ implica que el alcohol no se restringe en el hogar, $<<1>>$ implica que el alcohol sí se restringe en el hogar.

Tiempo que dispone al día, en promedio, la persona para cenar en su hogar durante la semana (multinomial): $<<0>>$ si la persona no cena, $<<1>>$ si la persona no tiene tiempo, $<<2>>$ si la persona se demora entre 15 a 30 minutos en cenar, $<<3>>$ entre 45 minutos y una hora y cuarto, $<<4>>$ entre una hora y media y dos horas, $<<5>>$ más de dos horas.

Rango del número de personas que viven en el hogar del encuestado (multinomial): $<<1>>$ implica que en el hogar viven entre 1-2 personas, $\langle<2>>$ entre 3-4 personas, $<<3>>, 50$ más personas.

Frecuencia del consumo de carne en el hogar (multinomial), $<<0>>$ implica que en el hogar no se consume carne, $<<1>>$ significa que en hogar se consume carne diariamente, $<<2>>$ se come carne $2-3$ veces a la semana, $<<3>>$ una vez a la semana, $<<4>>$ ocasionalmente. 


\section{TABLA 2}

Resultados de los modelos de regresión logit ordinal generado para medir la satisfacción con la alimentación de las personas de la zona Centro Sur de Chile ${ }^{\text {. }}$

\begin{tabular}{|c|c|c|c|c|}
\hline & Muestra total & $\mathrm{ABC} 1$ & $\mathrm{C} 2$ & $C 3, D$ y $E$ \\
\hline$Y^{2} b$ & $\begin{array}{c}-2,619^{* *} \\
(4,179)\end{array}$ & $\begin{array}{c}-5,853^{* * *} \\
(7,947)\end{array}$ & $\begin{array}{c}-5,389^{* * *} \\
(23,773)\end{array}$ & $\begin{array}{c}-4,778^{* *} \\
(6,477)\end{array}$ \\
\hline$Y^{3} b$ & $\begin{array}{c}-1,048 \\
(0,671)\end{array}$ & $\begin{array}{c}-4,098^{* *} \\
(3,975)\end{array}$ & $\begin{array}{c}-3,658^{* * *} \\
(12,223)\end{array}$ & $\begin{array}{l}-3,221^{*} \\
(3,040)\end{array}$ \\
\hline$Y^{4} b$ & $\begin{array}{c}0,861 \\
(0,455)\end{array}$ & $\begin{array}{l}-1,623 \\
(0,639)\end{array}$ & $\begin{array}{l}-1,644^{*} \\
(2,756)\end{array}$ & $\begin{array}{l}-1,352 \\
(0,559)\end{array}$ \\
\hline \multicolumn{5}{|l|}{ Variables explicativas } \\
\hline Gén= 1 & $\begin{array}{c}-0,610^{* * *} \\
(6,729)\end{array}$ & & & $\begin{array}{c}-0,922^{*} \\
(3,114)\end{array}$ \\
\hline Gén= 2 & $0^{c}$ & & & $0^{c}$ \\
\hline Mem-hogar= 1 & & & $\begin{array}{c}-1,135 \\
(2,683)\end{array}$ & $\begin{array}{c}-1,766^{* *} \\
(4,940)\end{array}$ \\
\hline Mem-hogar $=2$ & & & $\begin{array}{l}-0,702^{*} \\
(2,731)\end{array}$ & $\begin{array}{l}-0,064 \\
(0,015)\end{array}$ \\
\hline Mem-hogar $=3$ & & & $\mathrm{O}^{\mathrm{c}}$ & $\mathrm{O}^{\mathrm{c}}$ \\
\hline Per-Salud = 1 & $\begin{array}{c}-2,422^{* * *} \\
(22,375)\end{array}$ & $\begin{array}{c}-2,498^{* *} \\
(6,025)\end{array}$ & $\begin{array}{c}-1,816^{* *} \\
(4,212)\end{array}$ & $\begin{array}{c}-2,465^{* * *} \\
(7,541)\end{array}$ \\
\hline Per-Salud $=2$ & $\begin{array}{c}-1,798^{* * *} \\
19,769)\end{array}$ & $\begin{array}{c}-2,685^{* * *} \\
(13,825)\end{array}$ & $\begin{array}{l}-1,303^{*} \\
(3,509)\end{array}$ & $\begin{array}{c}-1,885^{* *} \\
(5,502)\end{array}$ \\
\hline Per-Salud $=3$ & $\begin{array}{c}-1,178 * * * \\
(9,999)\end{array}$ & $\begin{array}{c}-1,688^{* * *} \\
(7,275)\end{array}$ & $\begin{array}{l}-0,784 \\
(1,316)\end{array}$ & $\begin{array}{l}-0,396 \\
(0,227)\end{array}$ \\
\hline Per-Salud= 4 & $0^{c}$ & $0^{c}$ & $0^{c}$ & $\mathrm{O}^{\mathrm{c}}$ \\
\hline Tiempo-Cena-Sem $=0$ & $\begin{array}{c}-2,197^{* * *} \\
(8,456)\end{array}$ & $\begin{array}{c}-3,157^{* *} \\
(5,209)\end{array}$ & & \\
\hline Tiempo-Cena-Sem= 1 & $\begin{array}{c}-1,822^{* *} \\
(6,281)\end{array}$ & $\begin{array}{c}-3,284^{* *} \\
(5,685)\end{array}$ & & \\
\hline Tiempo-Cena-Sem = 2 & $\begin{array}{c}-2,033^{* * *} \\
(7,731)\end{array}$ & $\begin{array}{c}-3,622^{* * *} \\
(7,068)\end{array}$ & & \\
\hline Tiempo-Cena-Sem= 3 & $\begin{array}{c}-1,909^{* *} \\
6,386)\end{array}$ & $\begin{array}{c}-3,318^{* *} \\
(5,974)\end{array}$ & & \\
\hline Tiempo-Cena-Sem = 4 & $\begin{array}{c}-1,432 \\
(2,648)\end{array}$ & $\begin{array}{c}-4,432^{* * *} \\
(8,359)\end{array}$ & & \\
\hline Tiempo-Cena-Sem $=5$ & $0^{c}$ & $0^{c}$ & & \\
\hline Frec-Carne $=0$ & $\begin{array}{c}0,348 \\
(0,359)\end{array}$ & $\begin{array}{c}0,600 \\
(0,237)\end{array}$ & $\begin{array}{c}1,084 \\
(1,116)\end{array}$ & \\
\hline Frec-Carne $=1$ & $\begin{array}{l}1,583^{* * *} \\
(8,000)\end{array}$ & $\begin{array}{c}1,954^{*} \\
(3,227)\end{array}$ & $\begin{array}{c}1,961^{*} \\
(3,778)\end{array}$ & \\
\hline Frec-Carne $=2$ & $\begin{array}{c}0,109 \\
(0,072)\end{array}$ & $\begin{array}{c}0,690 \\
(0,645)\end{array}$ & $\begin{array}{l}-0,359 \\
(0,215)\end{array}$ & \\
\hline Frec-Carne $=3$ & $\begin{array}{c}0,183 \\
(0,166)\end{array}$ & $\begin{array}{c}0,300 \\
(0,114)\end{array}$ & $\begin{array}{l}-0,596 \\
(0,509)\end{array}$ & \\
\hline Frec-Carne $=4$ & $\mathrm{O}^{c}$ & $0^{c}$ & $0^{c}$ & \\
\hline Frec-Com-Rap $=0$ & $\begin{array}{l}1,564^{* *} \\
(4,087)\end{array}$ & & & $\begin{array}{c}0,968 \\
(0,616)\end{array}$ \\
\hline Frec-Com-Rap= 1 & $\begin{array}{l}1,941^{* *} \\
(6,028)\end{array}$ & & & $\begin{array}{c}1,173 \\
(0,905)\end{array}$ \\
\hline Frec-Com-Rap = 2 & $\begin{array}{c}2,161^{* * *} \\
(7,958)\end{array}$ & & & $\begin{array}{c}1,194 \\
(0,941)\end{array}$ \\
\hline Frec-Com-Rap= 3 & $\begin{array}{c}2,255^{* * *} \\
(8,095)\end{array}$ & & & $\begin{array}{l}2,611^{*} \\
(3,380)\end{array}$ \\
\hline Frec-Com-Rap= 4 & $\mathrm{O}^{\mathrm{c}}$ & & & $\mathrm{O}^{\mathrm{c}}$ \\
\hline Res-alcohol=0 & $\begin{array}{c}-0,527^{* *} \\
(5,073)\end{array}$ & $\begin{array}{c}-1,361^{* * *} \\
(9,784)\end{array}$ & & $\begin{array}{c}-1,526^{* * *} \\
(8,246)\end{array}$ \\
\hline Res-alcohol= 1 & $0^{c}$ & $0^{c}$ & & $0^{c}$ \\
\hline
\end{tabular}

a Variables significativas al nivel ${ }^{*} \mathrm{P}<0.10,{ }^{* *} \mathrm{P}<0.05$, ${ }^{* * *} \mathrm{P}<0.01$ basados en el estadístico de Wald. Los valores entre paréntesis corresponden al estadístico de Wald.

b Valor del parámetro umbral o límite (cut parameter). Hay tres parámetros umbral porque hay cuatro categorías de respuesta para la variable dependiente de

"satisfacción con la alimentación" (Satis).

c Este parámetro se establece en cero porque es redundante. Corresponde a la categoría de comparación de cada variable explicativa en cada modelo. 
mayor proporción de encuestados siempre desayuna (71,2\%), almuerza (50\%) y cena $(43,0 \%)$ en su hogar. Aproximadamente el $30 \%$ no cena y reemplaza esta comida por una más ligera (once). Fue superior la proporción de personas del NSE ABC1 $(P=0,042)$ que siempre desayuna en su hogar $(77,4 \%)$, caso contrario en el NSE C2 $(63,9 \%)$. La mayoría de los encuestados dispone de $15-30$ y de $45-75$ minutos para desayunar $(63,9 \%)$ y cenar $(51,6 \%)$, sin diferencias significativas según el NSE del encuestado $(P>0.1)$.

Resultados de los modelos logit ordinales

La tabla 2 muestra los resultados de los modelos logit ordinales generados para la satisfacción con la alimentación (Satis-Alim) correspondientes a la muestra total y a los NSE en estudio. Tanto en la muestra total como en las submuestras según NSE el ajuste de los modelos fue significativo al nivel $\mathrm{P}<0,001$ para el -2 log de la verosimilitud, con valores de pseudo R2 de Nagelkerke mayores que 0,3 (tabla 3). En la muestra total, considerando el signo de los coeficientes y las categorías de comparación de las variables que resultaron significativas en el modelo, se obtuvo que la probabilidad de que una persona tenga una alta probabilidad de satisfacción con su alimentación aumenta si consume carne diariamente $(\beta=1,583)$ respecto de aquellos que consumen este alimento ocasionalmente. El mismo efecto se observa si la persona nunca $(\beta=1,564)$, casi nunca $(\beta=1,941)$, ocasionalmente $(\beta=2,161)$ o generalmente $(\beta=2,255)$ come en locales de comida rápida, respecto de aquellos que siempre comen en estos locales. Por el contrario, la probabilidad de una alta satisfacción con la alimentación disminuye si la persona es de género masculino $(\beta=-0,610)$. Lo mismo es esperable si la auto percepción de la salud es muy mala o regular $(\beta=-2,422)$, buena $(\beta=-1,798)$ o muy buena $(\beta=-1,178)$, respecto de aquellos que tienen una auto percepción excelente de su salud. Asimismo, se reduce la probabilidad de una alta satisfacción con la alimentación si la persona no cena $(\beta=-2,197)$, no tiene tiempo para cenar $(\beta=-1,822)$, dispone de 15 a 30 minutos para esta comida $(\beta=$ $-2,033)$ o de entre 45 minutos a una hora y media $(\beta=-1,909)$, respecto de los participantes que pueden disponer de más de dos horas para cenar. Finalmente, en la muestra total disminuye la probabilidad de una alta satisfacción con la alimentación, si en el hogar del participante no se restringe el consumo de alcohol $(\beta=-0,527)$.

En el NSE ABC1 se obtuvo que la probabilidad de una alta satisfacción con la alimentación se incrementa si la persona consume carne en forma diaria $(\beta=1,954)$. Contrariamente, ésta disminuye si la auto percepción de la salud es muy mala o regular $(\beta=-2,498)$, buena $(\beta=-2,285)$ o muy buena $(\beta=$ $-1,688)$, respecto de aquellos que tienen una auto percepción excelente de su salud. Similar resultado se observó si la persona no cena $(\beta=-3,157)$, no tiene tiempo para cenar $(\beta=$ $-3,284)$, dispone de 15 a 30 minutos $(\beta=-3,622)$, de entre 45 minutos a una hora y media $(\beta=-3,318)$, o puede disponer de una hora y media a dos horas $(\beta=-4,432)$, respecto de los participantes que disponen de más de dos horas para cenar. En esta submuestra, también disminuye la probabilidad de una alta satisfacción con la alimentación, si no se restringe el consumo de alcohol $(\beta=-1,361)$ en el hogar. En la submuestra correspondiente al NSE C2, aumenta la probabilidad de una alta satisfacción con la alimentación si la persona consume carne diariamente $(\beta=1,961)$, pero ésta disminuye si el grupo familiar está compuesto por 3 a 4 integrantes $(\beta=-0,702)$ respecto de aquellos con familias con 5 o más integrantes. Lo mismo ocurre si la auto percepción de la salud es muy mala o regular $(\beta=-1,816)$ o si es buena $(\beta=-1,303)$, respecto de aquellos que tienen una auto percepción excelente de salud. En la submuestra de participantes de los NSE C3, D y E tomados en conjunto, la probabilidad de una alta satisfacción con la alimentación se incrementa si generalmente comen en locales de comida rápida $(\beta=2,611)$, respecto de aquellos que lo hacen siempre. Por el contrario, ésta disminuye si la persona es hombre $(\beta=-0,922)$, si el grupo familiar está conformado por una o dos personas $(\beta=-1,766)$, si la auto percepción de la salud es muy mala o regular $(\beta=-2,465)$ o buena $(\beta=-1,885)$ respecto de aquellos que tienen una auto percepción excelente de su salud $y$, si en el hogar no se restringe el consumo de alcohol $(\beta=-1,526)$.

\section{DISCUSION}

Los resultados de este estudio exploratorio permiten sugerir que disponer de mayores recursos económicos, asociados a un NSE más alto, aumenta la satisfacción con la alimentación. Sin embargo, un resultado destacable corresponde a las variables que resultaron explicativas de la satisfacción con la alimentación en la muestra total y en los distintos NSE

Al respecto, la única variable que resultó significativa para las cuatro muestras fue la auto percepción de la salud, disminuyendo el grado de satisfacción si ésta es mala, regular o buena. Esto permitiría confirmar la relación entre la alimentación y la salud $(13,24)$, así como también la asociación entre una mejor salud y un mayor nivel de satisfacción con la alimentación $(3,10)$.

Otro dominio de la vida que se relaciona con la satisfacción con la alimentación es la familia, a través del tiempo disponible para cenar en el hogar durante la semana en la muestra total y en el NSE ABC1 y, mediante el número de integrantes del grupo familiar en el NSE C2 y en la submuesta formada por los NSE C3, D y E. La mayor satisfacción con la alimentación en las personas del NSE ABC1 si disponen de más dos horas para cenar en su hogar en la semana, se relacionaría

\section{TABLA 3}

Evaluación de la bondad de ajuste del modelo logit ordinal generado para medir la satisfacción con la alimentación de personas de distinto NSE en la zona Centro Sur de Chilea.

\begin{tabular}{|c|c|c|c|c|}
\hline Estadístico & Muestra total & $A B C 1$ & $\mathrm{C} 2$ & $C 3, D, E$ \\
\hline $\mathrm{R}_{\text {adj. }}$ de Nagelkerke ${ }^{\mathrm{b}}$ & 0,346 & 0,444 & 0,353 & 0,440 \\
\hline$-2 \log$ de verosimilitud & 716,558 & 250,889 & 235,509 & 158,958 \\
\hline
\end{tabular}

a Modelos significativos al nivel *** $\mathrm{P}<0.001$ para la prueba -2 log de verosimilitud.

${ }^{b}$ El $R^{2}$ de Nagelkerke es un estadístico proxy del coeficiente de determinación (Pseudo- $R^{2}$ ) en el modelo logit. 
con la interacción social con la familia en torno a los alimentos $(3,8-10)$. Al respecto, es posible sugerir que esto se debe a que la cena constituye en muchos hogares chilenos la única oportunidad en que toda la familia come reunida, puesto que el régimen de jornada escolar completa imperante en Chile exige que los niños deban almorzar en su lugar de estudios desde temprana edad

Por lo tanto, una cena distendida permitiría a los padres interactuar con sus hijos luego de sus respectivas jornadas laborales y escolares. Si se considera que la frecuencia de comidas en familia se relaciona positivamente con la cohesión familiar (25) y con hábitos alimentarios más saludables, particularmente en adolescentes (26), la cena en familia debería ser una práctica habitual en todos los hogares chilenos y promovida por las autoridades de salud. La menor satisfacción de las personas del NSE C2 y de la submuestra compuesta por los NSE C3, D y E, si sus familias tienen menos de cinco integrantes, concordaría con la mayor satisfacción con la alimentación detectada en Europa si las personas preparan sus alimentos y comen en compañía de familiares (14). Esto sería más crítico en la submuestra formada por los NSE más bajos, en que la satisfacción con la alimentación disminuye si el núcleo familiar está formado sólo por una o dos personas, en los cuales es esperable que se coma en solitario con alta frecuencia. No obstante, estos resultados contrastan con los obtenidos en un estudio previo realizado con una muestra de personas de origen Mapuche en la Región de La Araucanía, en que se obtuvo un aumento de la satisfacción con la alimentación en la medida que viven menos personas en el hogar (7).

Adicionalmente, las diferencias en la satisfacción con la alimentación según el NSE, concuerda con las diferencias nutricionales y el tipo de alimentos consumidos entre personas de altos y bajos ingresos (13-17). En efecto, el aumento de la satisfacción con la alimentación en la muestra total y en los NSE ABC1 y C2 si comen carne en forma diaria, se contrapone con el aumento de la satisfacción con su alimentación en la submuestra formada por los NSE C3, D y E, si generalmente comen en locales de comida rápida. Al respecto, es posible sugerir que ambas variables están dando cuenta de que la satisfacción con la alimentación no sólo se vincula con la salud, sino que además se asocia al significado hedónico que tienen los alimentos (8-10). El consumo diario de carne para los de NSE más altos implicaría el acceso a alimentos de mayor calidad, en concordancia con los gustos y preferencias de la persona, sin limitaciones de precio. Es posible sugerir que comer frecuentemente en locales de comida rápida, ubicados en patios de comida de centros comerciales, aumenta la satisfacción con la alimentación de las personas de los NSE más bajos al estar comiendo el mismo tipo de comida que consumen personas de NSE más altos en este tipo de locales, sobre todo los fines de semana. No obstante, este comportamiento es congruente con los resultados de estudios que indican que las personas con menor capacidad de gasto poseen dietas de menor calidad, caracterizadas por una alta ingesta de energía proveniente de alimentos altos en grasa (15). Este resultado es preocupante desde el punto de vista de la nutrición y de la salud pública, debido a que las comidas en restaurantes y, particularmente, en locales de comida rápida tienen un impacto negativo en la salud (28), hábito que ha ido en aumento tanto en los países desarrollados como en las naciones en vías de desarrollo (27, 28). Paralelamente, se deben destacar las diferencias geográficas, debido a que en un estudio previo realizado en la Región Metropolitana de Santiago, se observó una disminución en la satisfacción con la alimentación asociada a una mayor frecuen- cia de consumo de alimentos en locales de comida rápida, por la presión del escaso tiempo disponible para comer (9).

La disminución en la satisfacción con la alimentación si no se restringe el consumo de alcohol en la muestra total, NSE $A B C 1$ y en la submuestra formada por los NSE C3, D y E, se relaciona con el hecho de que la mayor parte de los chilenos bebe en forma intermitente pero excesiva, lo que sitúa a los bebedores chilenos en una situación de elevado riesgo no sólo de problemas relativos al consumo de alcohol (trastorno por dependencia de alcohol), sino de un aumento de la incidencia de más de 40 patologías potenciales, la mayoría de ellas de tipo crónico, además de traumatismos y accidentes (29). Esto resulta un problema mayor para la submuestra formada por los NSE C3, D y E, debido a que la mayor prevalencia de problemas por abuso o dependencia de alcohol se encuentra en los NSE bajos (29). Esto no sólo afecta al individuo que abusa o tiene dependencia del alcohol, sino que también a sus familias. Finalmente, la menor satisfacción con la alimentación en hombres concuerda con un estudio previo en el sur de Chile (8), lo que podría estar relacionado con la menor preocupación por el cuidado de la salud respecto de las mujeres (30).

Los resultados de este estudio sirven de insumo para las autoridades de Salud, que deberían reforzar la promoción de la relación entre la alimentación y la salud, no solo en lo referente a la frecuencia de consumo en locales de comida rápida, sino que también en la frecuencia de consumo de frutas y verduras, puesto que en la muestra total la frecuencia de consumo de estos alimentos no cumple en todos los casos con las recomendaciones de la Organización Mundial de la Salud. La muestra obtenida a través los criterios de inclusión en la muestra y del método de muestreo utilizado, presenta una composición similar al país en cuanto a género, zona de residencia, edad y tamaño de la familia (31). La principal discrepancia corresponde al nivel socioeconómico (30\% correspondiente al estrato alto y medio-alto), puesto que en el país la proporción de personas pertenecientes al estrato ABC1 es de aproximadamente $10 \%$ (21), constituyendo esto la principal limitación del estudio. Por lo tanto, futuros estudios deberán abordar las variables que afectan la satisfacción con la alimentación en muestras representativas de la realidad socioeconómica del país, y que evalúen la influencia del NSE y del gasto mensual destinado a alimentos en conjunto.

\section{RESUMEN}

Con el objetivo de identificar variables que influyen en la satisfacción con la alimentación en personas pertenecientes a distintos niveles socioeconómicos (NSE) del sur de Chile, se aplicó una encuesta a 400 personas residentes en las principales ciudades de esta parte del país. El cuestionario incluyó la escala SWFL (Satisfaction with Food-related Life) y el Indice de Calidad de Vida relativo a la Salud (ICVRS). Se consultaron características demográficas y hábitos alimentarios. Se plantearon cuatro modelos logit ordinales, en los cuales la variable dependiente fue la satisfacción con la alimentación, considerando la muestra total y tres submuestras: estratos alto y medio-alto ( $A B C 1)$, medio-medio (C2) y en conjunto los niveles medio-bajo (C3), bajo (D) y muy bajo (E). Los cuatro modelos resultaron significativos $(p<0,001)$, pero difirieron en las variables explicativas significativas. Independientemente del NSE, la satisfacción con la alimentación disminuye si la persona no tiene una buena auto percepción de su salud. Otras variables que influyen en la satisfacción con la alimentación, que difirieron según el NSE, corresponden al género, composición de la familia, tiempo disponible para cenar en familia, 
frecuencia de consumo de carne y en locales de comida rápida y, la restricción del consumo de alcohol.

Palabras clave: Satisfacción con la alimentación, niveles socioeconómicos, salud.

Agradecimientos: Los resultados corresponden al Proyecto Fondecyt 1100611.

\section{BIBLIOGRAFÍA}

1. Grunert K, Dean D, Raats M, Nielsen N, Lumbers M. A measure of satisfaction with food-related life, Appetite 2007; 49(2): 486-93.

2. Schnettler $B$, Miranda $H$, Sepúlveda J, Denegri M, Mora $M$, Lobos $G$. Satisfaction with life and food-related life in persons of the mapuche ethnic group in southern Chile. A comparative analysis using logit and probit models, J Happiness Stud. 2012; 13(2): 225-46.

3. Seo S, Cho M, Kim Y, Ahn J. The Relationships among satisfaction with food-related life, depression, isolation, social support, and overall satisfaction of life in elderly South Koreans, J Korean Diet Assoc. 2013; 19(2): 159-60.

4. Schnettler B, Miranda $H$, Sepúlveda J, Denegri M, Mora $M$, Lobos G, Grunert KG. Psychometric properties of the satisfaction with food-related life scale: application in southern Chile, J Nutr Educ Behav. 2013; 45(5): 443-9.

5. Schnettler B, Miranda H, Sepúlveda J, Mora M, Lobos G, Denegri M. Satisfacción con la vida en personas de etnia Mapuche residentes en la Región Metropolitana, Chile. Un análisis comparativo con modelos Logit y Probit. Suma Psicol. 2013; 20(1): 69-82.

6. Schnettler B, Miranda $H$, Sepúlveda J, Denegri M, Mora $M$, Lobos $G$. Satisfacción con la alimentación en personas Mapuche en la Región Metropolitana de Santiago, Chile, Arch Latinoam Nutr. 2011; 61(2): 172-82.

7. Schnettler B, Mora M, Miranda H, Sepúlveda J, Denegri $M$, Lobos $G$. Satisfacción con la alimentación en personas Mapuche en la Región de La Araucanía, Chile. Rev Chil Nutr. 2012; 39(1): 18-29.

8. Schnettler B, Mora M, Mills N, Miranda H, Sepúlveda J, Denegri $M$, Lobos $G$. Tipologías de consumidores según el estilo de vida en relación a la alimentación: un estudio exploratorio en el sur de Chile, Rev Chil Nutr. 2012, 39(4): 165-72.

9. Schnettler B, Peña JP, Mora M, Miranda H, Sepúlveda J, Denegri $M$, Lobos $G$. Estilos de vida en relación a la alimentación y hábitos alimentarios dentro y fuera del hogar en la Región Metropolitana de Santiago, Chile. Nutr Hosp. 2013; 28(4):1266-73.

10. Dean M, Grunert K, Raats M, Nielsen NA, Lumbers M, Food in Later Life Team. The impact of personal resources and their goal relevance on satisfaction with food-related life among the elderly, Appetite 2008; 50(2-3): 308-15.

11. Lavers T. Asking people what they want or telling them what they 'need'? Contrasting a theory of human need with local expressions of goals. Soc Indic Res. 2008; 86: 129-47.

12. Guillen-Royo M. Consumption and subjective wellbeing: exploring basic needs, social comparison, social integration and hedonism in Peru, Soc Indic Res. 2008; 89(3): 535-55.

13. Cummins RA. (2000). Personal income and subjective wellbeing: A review, J Happiness Stud. 2000; 1(2): 133-58.

14. Story $M$, Kaphingst $K$, Robinson-O'Brien $R$, Glanz $K$. Healthy food and eating environments. Policy and envi- ronmental approaches, Annu Rev Publ Health 2008; 29: 253-72.

15. French S, Wall M, Mitchell N. Household income differences in food sources and food items purchased. Int J Behav Nutr Phy. 2010; 7: 77.

16. Giskes $K$, van Lenthe F, Avendano-Pabon M, Brug J. A systematic review of environmental factors and obesogenic dietary intakes among adults. Are we getting close to understanding obesogenic environments?, Obes Rev. 2011; 12: 95-106.

17. Fazal S, Valdettaro PM, Friedman J, Basquin C, Pietzsch $S$. Towards improved food and nutrition security in Sindh Province, Pakistan, IDS Bull-I Dev Stud. 2013; 44(3): 21-30.

18. Fernández A. Investigación y técnicas de mercado. Primera edición. Madrid: Esic; 2002.

19. Hennessy C, Moriarty D, Zack M, Scherr P, Brackbill R. Measuring health-related quality of life for public health surveillance, Publ Health Rep. 1994; 109: 665-72.

20. INE. Metodología VI Encuesta de presupuestos familiares 2006-2007. Resultados Encuestas de Presupuestos familiares. Nov. 2006-oct. 2007. Disponible en: http://www.ine. cl/canales/chile_estadistico/encuestas_presupuestos_familiares/2008/resultados_EPF_2006_2007_080708a.pdf [Conectado en diciembre de 2008].

21. Adimark. Mapa socioeconómico de Chile. 2004. Disponible en http://www.adimark.cl/medios/estudios/ informe_mapa_socioeconomico_de_chile.pdf [Conectado en octubre de 2005].

22. Hair J, Anderson R, Tatham R, Black W. Análisis multivariante. Otero. $5^{\text {a }}$ ed. Madrid: Prentice Hall Internacional. Inc.; 1999.

23. Greene WH. Análisis econométrico. $3^{a}$ ed. Madrid: Prentice-Hall; 1999.

24. Chen M-F. The joint moderating effect of health consciousness and healthy lifestyle on consumers' willingness to use functional foods in Taiwan, Appetite 2011; 57(1): 253-62.

25. Welsh E, French S, Wall M. Examining the Relationship Between Family Meal Frequency and Individual Dietary Intake: Does Family Cohesion Play a Role?, J Nutr Educ Behav. 2011; 43(4): 229-35.

26. Burgess-Champoux TL, Larson N, Neumark-Sztainer D, Hannan PJ, Story M. Are family meal patterns associated with overall diet quality during the transition from early to middle adolescence?, J Nutr Educ Behav. 2009; 41: 79-86.

27. Vandevijvere $S$, Lachat $C$, Kolsteren $P$, Van Oyen H. Eating out of home in Belgium: current situation and policy implications, Br J Nutr. 2009; 102: 921-8.

28. Bezerra IN, Sichieri R. Characteristics and spending on out-of home eating in Brazil. Rev Saude Publica 2010; 44: 221-9.

29. Ministerio de Salud de Chile. Encuesta Nacional de Salud ENS Chile. 2009-2010. Disponible en: http://www. minsal.gob.cl/portal/docs/page/minsalcl/g_home/submenu_portada_2011/ens2010.pdf. [Conectado el 24 de enero de 2012].

30. Wyness L, Weichselbaum E, O'Connor A, Williams EB, Benelam B, Riley $H$, Stanner S. Red meat in the diet: an update, Nutr Bull 2011; 36(1): 34-77.

31. Instituto Nacional de Estadísticas (INE). Censo 2002. Resultados Volumen I. Población; País-Región. Santiago, Chile. 2003. 